\title{
ON THEOREMS OF BEURLING AND HARDY FOR THE EUCLIDEAN MOTION GROUP
}

\author{
Rudra P. SARKAR AND Sundaram ThangaVelu
}

(Received September 22, 2003, revised June 7, 2004)

\begin{abstract}
We establish an analogue of Beurling's uncertainty principle for the group Fourier transform on the Euclidean motion group. We also prove the most general version of Hardy's theorem on it which characterises functions on the motion group that are controlled by the heat kernel associated to the Laplacian of the Euclidean space.
\end{abstract}

1. Introduction. A cute little theorem of Beurling on Fourier transform pairs which was published by Hörmander [7] says that for any nontrivial function $f$ in $L^{2}(\boldsymbol{R})$, the function $f(x) \hat{f}(y)$ is never integrable on $\boldsymbol{R}^{2}$ with respect to the measure $e^{|x y|} d x d y$. A far-reaching generalisation of this result has been recently proved by Bonami et al. [1]. Let

$$
\hat{f}(y)=(2 \pi)^{-n / 2} \int_{\boldsymbol{R}^{n}} e^{-i(x, y)} f(x) d x
$$

stand for the Fourier transform of a function $f$ on $\boldsymbol{R}^{n}$. Then we have:

THEOREM 1.1 (Bonami-Demange-Jaming). Let $f \in L^{2}\left(\boldsymbol{R}^{n}\right)$ and suppose that for some $N \geq 0$

$$
\int_{\boldsymbol{R}^{n}} \int_{\boldsymbol{R}^{n}} \frac{|f(x)|}{(1+|x|)^{N}} \frac{|\hat{f}(y)|}{(1+|y|)^{N}} e^{|(x, y)|} d x d y<\infty .
$$

Then $f=0$ whenever $N \leq n$. If $N>n$, then the above holds if and only if $f$ can be written as

$$
f(x)=P(x) e^{-(A x, x) / 2},
$$

where $A$ is a real positive definite matrix and $P$ is a polynomial of degree $\leq(N-n) / 2$.

Some attempts to generalise this result to group Fourier transforms on certain Lie groups have already been made. Unlike the Euclidean case functions $f$ and their Fourier transforms $\hat{f}$ live on different sets which spoils the elegance of the above result in other contexts.

In this paper we formulate and prove an analogue of Theorem 1.1 for the Fourier transform on Euclidean motion groups $M(n)$.

Let $M(n)$ be the semidirect product of $\boldsymbol{R}^{n}$ with $K=S O(n)$. The group law is given by

$$
(x, k)\left(y, k^{\prime}\right)=\left(x+k \cdot y, k k^{\prime}\right), \quad x, y \in \boldsymbol{R}^{n}, \quad k, k^{\prime} \in K .
$$

2000 Mathematics Subject Classification. Primary 43A80; Secondary 22E.

Key words and phrases. Beurling's Theorem, Hardy's Theorem, Motion group.

The authors are grateful to an anonymous referee for many suggestions. They are also thankful to Ms. Asha Lata for her excellent job of typing the manuscript. 
Let $M=S O(n-1)$ be considered as a subgroup of $K$ leaving the point $e_{1}=(1,0,0, \ldots, 0)$ fixed. Then all the irreducible unitary representations of $M(n)$ relevant for the Plancherel theorem are parametrised (upto unitary equivalence) by pairs $(\lambda, \sigma)$ where $\lambda>0$ and $\sigma \in \hat{M}$, the unitary dual of $M$. (When $n=2$ there is only one parameter namely, $\lambda>0$.) The group Fourier transform of $f \in L^{1}(M(n))$ is then defined by

$$
\hat{f}(\lambda, \sigma)=\int_{M(n)} f(x, k) \pi_{\lambda, \sigma}(x, k) d x d k
$$

where $\pi_{\lambda, \sigma}(x, k)$ is the irreducible unitary representation of $M(n)$ associated to the parameter $(\lambda, \sigma)$. These representations are realised on certain Hilbert spaces which will be described in the following sections. Let $\|\hat{f}(\lambda, \sigma)\|_{\mathrm{HS}}$ stand for the Hilbert-Schmidt operator norm of $\hat{f}(\lambda, \sigma)$. As an analogue of Beurling's theorem we offer:

THEOREM 1.2. Let $f \in L^{1} \cap L^{2}(M(n))$ and assume that

$$
\int_{M(n)} \int_{0}^{\infty} \frac{\|\hat{f}(\lambda, \sigma)\|_{\mathrm{HS}}}{(1+\lambda)^{n}} \frac{|f(x, k)|}{(1+|x|)^{n}} e^{\lambda|x|} \lambda^{n-1} d \lambda d x d k<\infty
$$

for every $\sigma \in \hat{M}$. Then $f=0$.

Note that we have considered only the case $N=n$. When $n=2$ we can do slightly better. Let us write $\left(z, e^{i \varphi}\right)$ for the coordinates on $M(2), z \in \boldsymbol{R}^{2}$.

THEOREM 1.3. Let $f \in L^{1} \cap L^{2}(M(2))$ and assume that

$$
\int_{M(2)} \int_{0}^{\infty} \frac{\|\hat{f}(\lambda)\|_{\mathrm{HS}}}{(1+\lambda)^{3}} \frac{\left|f\left(z, e^{i \varphi}\right)\right|}{(1+|z|)^{3}} e^{\lambda|z|} \lambda d \lambda d z d \varphi<\infty .
$$

Then $f$ can be represented as

$$
f\left(z, e^{i \varphi}\right)=g\left(e^{i \varphi}\right) e^{-a|z|^{2}}
$$

for some $g \in L^{2}(T)$ and $a>0$.

In this theorem $T$ stands for the circle group which is identified with $S O(2)$. Let $\Delta_{n}$ be the standard Laplacian on $\boldsymbol{R}^{n}$ and let

$$
p_{t}^{n}(x)=(4 \pi t)^{-n / 2} e^{-|x|^{2} / 4 t}
$$

be the associated heat kernel. Then the final conclusion of the above theorem can be expressed in terms of the heat kernel $p_{t}^{n}(z)$ on $\boldsymbol{R}^{n}$. The original theorem of Beurling $(N=0)$ can be viewed as an uncertainty principle for the Fourier transform. The case $N=n+1$ is then a characterisation of the heat kernel $p_{t}(x)$ on $\boldsymbol{R}^{n}$. Theorem 1.1 is so strong that other 'uncertainty principles' like the theorems of Hardy, Gelfand-Shilov and Cowling-Price all follow from it. For example, a general form of Hardy's theorem for $\boldsymbol{R}^{n}$ in [15] says that if $f \in L^{1}\left(\boldsymbol{R}^{n}\right)$ satisfies

$$
|f(x)| \leq c(1+|x|)^{m} p_{s}^{n}(x), \quad|\hat{f}(y)| \leq c(1+|y|)^{m} e^{-t|y|^{2}}
$$


then $f=0$ whenever $s<t$ and when $s=t, f(x)=Q(x) p_{t}^{n}(x)$ where $Q$ is a polynomial of degree $\leq m$. It is clear that this result follows immediately from Theorem 1.1.

Even though we do not have the most general version of Beurling's theorem for $M(n)$, we do have a general version of Hardy's theorem.

THEOREM 1.4. Let $f \in L^{1}(M(n))$ satisfy the following two conditions:

$$
\begin{aligned}
|f(x, k)| & \leq c(1+|x|)^{N} p_{s}^{n}(x), \quad(x, k) \in M(n) \\
\|\hat{f}(\lambda, \sigma)\| & \leq c(1+\lambda)^{N} e^{-t \lambda^{2}}, \quad(\lambda, \sigma) \in \boldsymbol{R}_{+} \times \hat{M} .
\end{aligned}
$$

Then $f=0$ whenever $s<t$. When $s=t, f$ can be expressed as a finite linear combination of functions of the form

$$
P_{m, j}(x)\left(-\Delta_{n+2 m}\right)^{(j-m) / 2} p_{t}^{n+2 m}(x) g_{m j}(k)
$$

where $P_{m j}$ are solid harmonics of degree $m$ and $g_{m j}$ are certain bounded functions in $L^{2}(K)$.

In the above the fractional powers of the Laplacian are defined via the Fourier transform:

$$
\left(-\Delta_{n}\right)^{j / 2} f(x)=(2 \pi)^{-n / 2} \int_{R^{n}}|\xi|^{j} \hat{f}(\xi) e^{i x \cdot \xi} d \xi
$$

The range of $m$ and $j$ are restricted by the condition

$$
\left|P_{m j}(x)\right|\left|\left(-\Delta_{n+2 m}\right)^{(j-m) / 2} p_{t}^{n+2 m}(x)\right| \leq c(1+|x|)^{N} p_{t}^{n}(x) .
$$

The case $N=0, s<t$ of the above theorem is due to Sundari [14]. See the work of Eguchi et al. [3] for an analogue (again the case $N=0, s<t$ ) of the above for Cartan motion groups. Eguchi et al. [4] have also treated an $L^{p}$ version of the above result $(N=0, s<t)$. The above theorem with $N>0$ and $s=t$ is new.

It is possible to prove a refined version of the above theorem when $n=2$. Compare the following result with Hardy's theorem proved in [16] for the Euclidean Fourier transform. When $n=2$, the relevant representations $\pi_{\lambda}\left(z, e^{i \varphi}\right)$ of $M(2)$ are parametrised by $\lambda>0$ and realised on the same Hilbert space $L^{2}(T)$. Let $\left\{e_{k}(\theta)=e^{i k \theta}: k \in Z\right\}$ be the standard basis for $L^{2}(T)$.

THEOREM 1.5. Let $f \in L^{1}(M(2))$ satisfy the estimate

$$
\left|f\left(z, e^{i \varphi}\right)\right| \leq c p_{t}^{2}(z), \quad z \in \boldsymbol{R}^{2} .
$$

Further assume that for every $\lambda>0$, and $k, j \in \boldsymbol{Z}$

$$
\left|\left(\hat{f}(\lambda) e_{k}, e_{j}\right)\right| \leq c_{k j} \lambda^{|k-j|} e^{-t \lambda^{2}} .
$$

Then $f$ can be represented as

$$
f\left(z, e^{i \varphi}\right)=p_{t}^{2}(z)\left(\int_{\boldsymbol{R}^{n}} f\left(z, e^{i \varphi}\right) d z\right) .
$$

The plan of the paper is as follows. In the next section we collect relevant information from the representation theory of $M(n)$. We also recall a theorem of Gross and Kunze [6] which is needed for the proof of Theorem 1.2. All the results are proved in Sections 3 and 
4. For analogues of Hardy's theorem in the contexts of other groups we refer to [2], [11] and [15] and the references there.

2. Representation theory of $M(n)$. In this section we describe the unitary dual of $M(n)$. To enhance the readability of this paper we collect relevant information from the representation theory of $M(n)$. General references are the books of Folland [5] and Suguira [13]. We also need some results from the paper of Gross and Kunze [6].

First consider the case $M(2)$. We write the elements of $M(2)$ as $\left(z, e^{i \varphi}\right), z \in \boldsymbol{C}$ and $\varphi \in \boldsymbol{R}$. The group law is given by

$$
\left(z, e^{i \varphi}\right)\left(w, e^{i \theta}\right)=\left(z+e^{i \varphi} w, e^{i(\theta+\varphi)}\right) .
$$

For each $\lambda>0$ consider the representation $\pi_{\lambda}\left(z, e^{i \varphi}\right)$ realised on $L^{2}(T)$ :

$$
\pi_{\lambda}\left(z, e^{i \varphi}\right) g(\theta)=e^{i \operatorname{Re}\left(\lambda e^{-i \theta} z\right)} g(\theta-\varphi)
$$

where $g \in L^{2}(T)$. As shown in Sugiura [13] any infinite dimensional irreducible unitary representation of $M(2)$ is unitarily equivalent to $\pi_{\lambda}$ for some $\lambda>0$.

Next consider the case $M(n), n \geq 3$. As in the introduction we let $K=S O(n)$ and $M=S O(n-1)$. Given $\sigma \in \hat{M}$ realised on a Hilbert space $V_{\sigma}$ of dimension $d_{\sigma}$ consider the space $L^{2}(K, \sigma)$ consisting of functions $\varphi$ on $K$ taking values in $C^{d_{\sigma} \times d_{\sigma}}$, the space of $d_{\sigma} \times d_{\sigma}$ complex matrices, satisfying the condition

$$
\varphi(u k)=\sigma(u) \varphi(k), \quad u \in M, k \in K
$$

which are also square integrable on $K$ :

$$
\int_{K}\|\varphi(k)\|^{2} d k=\int_{K} \operatorname{tr}\left(\varphi(k)^{*} \varphi(k)\right) d k<\infty .
$$

Note that $L^{2}(K, \sigma)$ is a Hilbert space under the inner product

$$
(\varphi, \psi)=\int_{K} \operatorname{tr}\left(\varphi(k) \psi(k)^{*}\right) d k
$$

For each $\lambda>0$ and $\sigma \in \hat{M}$ we can define a primary representation $\pi_{\lambda, \sigma}$ of $M(n)$ on $L^{2}(K, \sigma)$ as follows. For $\varphi \in L^{2}(K, \sigma),(x, k) \in M(n)$,

$$
\pi_{\lambda, \sigma}(x, k) \varphi(u)=e^{i \lambda\left(u^{-1} \cdot e_{1}, x\right)} \varphi(u k), \quad u \in K .
$$

If $\varphi_{j}(k)$ are the column vectors of $\varphi \in L^{2}(K, \sigma)$ then $\varphi_{j}(u k)=\sigma(u) \varphi_{j}(k)$ for all $u \in M$. Therefore, $L^{2}(k, \sigma)$ can be written as the direct sum of $d_{\sigma}$ copies of $H(K, \sigma)$ which is defined to be the space of $\varphi: K \rightarrow C^{d_{\sigma}}$ which are square integrable and satisfy

$$
\varphi(u k)=\sigma(u) \varphi(k), \quad u \in M .
$$

It can be shown that $\pi_{\lambda, \sigma}$ restricted to $H(K, \sigma)$ is an irreducible unitary representation of $M(n)$. Moreover, any irreducible unitary representation of $M(n)$ which is infinite dimensional is unitarily equivalent to one and only one $\pi_{\lambda, \sigma}$. Finite dimensional irreducible unitary representations of $K$ also yield irreducible unitary representations of $M(n)$. As they do not appear in the Plancherel formula we neglect them. 
Given $f \in L^{1} \cap L^{2}(M(n))$ we define the group Fourier transform of $f$ by

$$
\hat{f}(\lambda, \sigma)=\int_{M(n)} f(x, k) \pi_{\lambda, \sigma}(x, k) d x d k .
$$

Note that $\hat{f}(\lambda, \sigma)$ is a Hilbert-Schmidt operator on $H(K, \sigma)$. We would like to think of the Fourier transform $\hat{f}$ as an operator on a fixed Hilbert space. To achieve this we simply take the direct sum of all the Hilbert spaces $L^{2}(K, \sigma)$. More precisely, let $L^{2}(K)^{\wedge}$ stand for the direct sum of $L^{2}(K, \sigma)$ as $\sigma$ varies over $\hat{M}$. Thus $L^{2}(K)^{\wedge}$ is the set of all functions $F$ on $\hat{M}$ such that $F(\sigma)$ belongs to $L^{2}(K, \sigma)$ for each $\sigma$ and

$$
\sum_{\sigma \in \hat{M}}\|F(\sigma)\|^{2}<\infty .
$$

The Hilbert space structure of $L^{2}(K)^{\wedge}$ is given by

$$
(F, G)=\sum_{\sigma \in \hat{M}}(F(\sigma), G(\sigma)) .
$$

For each $\lambda>0$ we can now define $\hat{f}(\lambda)$ as an operator on $L^{2}(K)^{\wedge}$ by setting

$$
(\hat{f}(\lambda) F)(\sigma)=\hat{f}(\lambda, \sigma) F(\sigma) .
$$

In the proof of Theorem 1.3 we make use of a result of Gross and Kunze [6] which shows that $L^{2}(K)^{\wedge}$ is isomorphic to $L^{2}(K)$. To state their result we need to set up some notation. Let $L(u)$ stand for the left regular representation of $M$ on $L^{2}(K)$ given by $L(u) \psi(k)=\psi\left(u^{-1} k\right)$. For any $\sigma \in \hat{M}$ consider the projection

$$
P_{\sigma}=d_{\sigma} \int_{M} \chi_{\sigma}(u) L(u) d u
$$

where $\chi_{\sigma}(u)=\operatorname{tr}(\sigma(u))$ is the character of $\sigma$. A vector lies in the range of $P_{\sigma}$ if and only if its translates under $L$ span a finite dimensional subspace in which $L$ decomposes into irreducible representations equivalent to $\sigma$.

Note that when $\psi \in L^{2}(K)$ the function

$$
\tilde{\psi}(\sigma, k)=\int_{M} \psi\left(u^{-1} k\right) \sigma(u) d u
$$

takes values in $L^{2}(K, \sigma)$ as can be easily checked. The generalised Fourier transform of a function $\psi \in C(K)$ is the function $U \psi \in L^{2}(K)^{\wedge}$ whose value at $\sigma \in \hat{M}$ is given by

$$
U \psi(\sigma)(k)=\tilde{\psi}(\sigma, k) .
$$

In [6] the following theorem has been proved:

THEOREM 2.1 (Gross and Kunze). The generalised Fourier transform $\psi \mapsto U \psi$ initially defined on $C(K)$ extends uniquely to a unitary operator between $L^{2}(K)$ onto $L^{2}(K)^{\wedge}$ which maps $P_{\sigma} L^{2}(K)$ onto $L^{2}(K, \sigma)$. 
For each $\lambda>0$ we can define $\hat{f}(\lambda)$ as an operator on $L^{2}(K)^{\wedge}$ and using the above unitary operator $U: L^{2}(K) \rightarrow L^{2}(K)^{\wedge}$ we can consider $U^{*} \hat{f}(\lambda) U$ as an operator on $L^{2}(K)$ which is unitarily equivalent to $\hat{f}(\lambda)$. We use this identification to obtain a formula for

$$
\sum_{\sigma \in \hat{M}}\|\hat{f}(\lambda, \sigma)\|_{\mathrm{HS}}^{2}
$$

which is crucial in the proof of Beurling's theorem 1.3. We remark that the Plancherel theorem for $M(n)$ takes the form

$$
c_{n} \int_{0}^{\infty}\left(\sum_{\sigma \in \hat{M}}\|\hat{f}(\lambda, \sigma)\|_{\mathrm{HS}}^{2}\right) \lambda^{n-1} d \lambda=\int_{M(n)}|f(x, k)|^{2} d x d k
$$

for a suitable constant $c_{n}$.

3. Hardy's theorem for $M(n)$. In this section we prove Theorems 1.4 and 1.5 . We first recall some results from the Euclidean harmonic analysis which will be needed in the proofs. First of all we make use of the Hecke-Bochner formula for the Fourier transform which states that if $f(x)=P(x) g(|x|)$ where $g$ is radial and $P$ is a solid harmonic of degree $m$ then

$$
(2 \pi)^{-n / 2} \int_{\boldsymbol{R}^{n}} f(x) e^{-i x \cdot \xi} d x=(-i)^{m} P(\xi) G(|\xi|)
$$

where $G(|\xi|)$ is the Fourier transform of the radial function $g(|x|)$ on $\boldsymbol{R}^{n+2 m} \cdot G(|\xi|)$ is also given as a Hankel transform of order $(n / 2+m-1)$. For these facts we refer to Stein-Weiss [12].

In the above, a solid harmonic of degree $m$ is a polynomial which is homogeneous of degree $m$ and harmonic. The set of all such polynomials will be denoted by $\mathcal{H}_{m}$ and the restrictions of elements of $\mathcal{H}_{m}$ to $S^{n-1}$ is denoted by $\mathcal{S}_{m}$. Members of $\mathcal{S}_{m}$ are called spherical harmonics of degree $m$. By choosing an orthonormal basis $\left\{Y_{m j} ; j=1,2, \ldots, d_{m}\right\}$ of $\mathcal{S}_{m}$ for each $m=0,1,2, \ldots$ we get an orthonormal basis for $L^{2}\left(S^{n-1}\right)$.

The Funk-Hecke formula deals with spherical harmonic expansions of zonal functions. If $a$ is a function on $(-1,1)$ this formula says that

$$
\int_{S^{n-1}} a\left(x^{\prime} \cdot y^{\prime}\right) Y_{m j}\left(y^{\prime}\right) d y^{\prime}=c_{m j} Y_{m j}\left(x^{\prime}\right) .
$$

The coefficients $c_{m j}$ are given in terms of ultraspherical polynomials. More precisely, let $G_{m}^{n / 2-1}(t)$ be ultraspherical polynomials of type $(n / 2-1)$. Then

$$
c_{m j}=\frac{\Gamma(m+1) \Gamma(n-2)}{\Gamma(m+n-2)} w_{n-2} \int_{-1}^{1} a(t) G_{m}^{n / 2-1}(t)\left(1-t^{2}\right)^{(n-3) / 2} d t .
$$

We refer to Muller [8] for this formula. In particular, taking $a(t)=e^{i \lambda r t}$, we have

$$
\int_{S^{n-1}} e^{i \lambda r x^{\prime} \cdot y^{\prime}} Y_{m j}\left(y^{\prime}\right) d y^{\prime}=c_{m j} Y_{m j}\left(x^{\prime}\right) \text {. }
$$


Using the Poisson integral representation of Bessel functions we can calculate $c_{m j}$ leading to the formula

$$
e^{i \lambda r x^{\prime} \cdot y^{\prime}}=\sum_{m=0}^{\infty} \sum_{j=1}^{d_{m}} \frac{J_{n / 2+m-1}(\lambda r)}{(\lambda r)^{n / 2-1}} Y_{m j}\left(x^{\prime}\right) Y_{m j}\left(y^{\prime}\right)
$$

where $J_{\alpha}(t)$ is the Bessel function of type $\alpha$.

Hecke-Bochner formula (3.1) leads to a simple description of $\hat{f}(\lambda, \sigma)$ when $f$ is of a special form.

LEMMA 3.1. Let $f \in L^{1}(M(n))$ be of the form $f(x, u)=P(x) g(|x|) h(u)$ where $P$ is a solid harmonic of degree $m$. Then for every $\lambda>0$ and $\sigma \in \hat{M}$ we have

$$
\hat{f}(\lambda, \sigma) \varphi(k)=\lambda^{m} G(\lambda) P\left(k^{-1} e_{1}\right) \int_{K} h(u) \varphi(k u) d u
$$

where $G(\lambda)$ is the $(n+2 m)$-dimensional Fourier transform of $g(|x|)$.

The lemma follows immediately from the definition of $\pi_{\lambda, \sigma}(x, u)$ in view of (3.1). In particular when we take

$$
f(x, u)=P(x)\left(-\Delta_{n+2 m}\right)^{(j-m) / 2} p_{t}^{n+2 m}(x) h(u)
$$

we see that

$$
\hat{f}(\lambda, \sigma) \varphi(k)=\lambda^{j} e^{-t \lambda^{2}} P\left(k^{-1} e_{1}\right) \int_{K} h(u) \varphi(k u) d u
$$

for every $\varphi \in H(K, \sigma)$. This formula will be used in the proof of Theorem 1.4.

We also need the following lemma on entire functions of order 2.

LEMMA 3.2. Let $F(z)$ be an entire function of a single complex variable $z$ which satisfies

$$
\begin{aligned}
& |F(z)| \leq c(1+|z|)^{N} e^{a|\operatorname{Im}(z)|^{2}}, \quad z \in \boldsymbol{C}, \\
& |F(x)| \leq c(1+|x|)^{N} e^{-a x^{2}}, \quad x \in \boldsymbol{R} .
\end{aligned}
$$

Then $F(z)=P(z) e^{-a z^{2}}$ where $P(z)$ is a polynomial of degree $\leq N$.

A proof of this lemma can be found in Sarkar [10]. Having collected all the results needed, we begin with a proof of Theorem 1.4. Note that we only need to consider the case $s=t$. For $\varphi, \psi \in H(K, \sigma)$ consider

$$
(\hat{f}(\lambda, \sigma) \varphi, \psi)=\int_{M(n)} \int_{K} f(x, u) e^{i \lambda k^{-1} e_{1} \cdot x}(\varphi(k u), \psi(k)) d k d x d u .
$$

Since the function $f$ satisfies the estimate

$$
|f(x, u)| \leq c(1+|x|)^{N} p_{t}^{n}(x)
$$


it is clear that the above integral (3.7) converges even if $\lambda \in \boldsymbol{C}$. Indeed,

$$
\begin{aligned}
\left|\int_{K} e^{i \lambda\left(k^{-1} e_{1} \cdot x\right)}(\varphi(k u), \psi(k)) d k\right| & \leq e^{|I m(\lambda) \| x|} \int_{K}|\varphi(k u) \| \psi(k)| d k \\
& \leq e^{|I m(\lambda) \| x|}\|\varphi\|\|\psi\| .
\end{aligned}
$$

Using this estimate and (3.8) in (3.7) we see that

$$
|(\hat{f}(\lambda, \sigma) \varphi, \psi)| \leq c(1+|\lambda|)^{N} e^{t|\operatorname{Im}(\lambda)|^{2}}\|\varphi\|\|\psi\| .
$$

Thus the function $\lambda \rightarrow(\hat{f}(\lambda, \sigma) \varphi, \psi)$ extends to an entire function of order 2 satisfying the estimate (3.9) for all $\lambda \in \boldsymbol{C}$. We are also given the estimate

$$
|(\hat{f}(\lambda, \sigma) \varphi, \psi)| \leq c(1+|\lambda|)^{N} e^{-t|\lambda|^{2}}\|\varphi\|\|\psi\|
$$

for all $\lambda \in \boldsymbol{R}$. Appealing to the complex analytic Lemma 3.2 we conclude that

$$
(\hat{f}(\lambda, \sigma) \varphi, \psi)=P_{\sigma}(\lambda, \varphi, \psi) e^{-t \lambda^{2}}
$$

where $P_{\sigma}(\lambda, \varphi, \psi)$ is a polynomial of degree atmost $N$.

It is now clear that $P_{\sigma}(\lambda, \varphi, \psi)$ is linear in $\varphi$ and $\psi$. If we write

$$
P_{\sigma}(\lambda, \varphi, \psi)=\sum_{j=0}^{N} a_{\sigma, j}(\varphi, \psi) \lambda^{j}
$$

then it can be easily shown that $a_{\sigma, j}(\varphi, \psi)$ are sesquilinear forms on $H(K, \sigma)$. Cauchy's formula together with the estimate (3.9) shows that $a_{\sigma, j}(\varphi, \psi)$ are actually bounded:

$$
\left|a_{\sigma, j}(\varphi, \psi)\right| \leq c_{\sigma, j}\|\varphi\|\|\psi\|
$$

where $c_{\sigma, j}$ are constants independent of $\varphi$ and $\psi$. Hence there are bounded linear operators $A_{\sigma, j}$ on $H(K, \sigma)$ such that

$$
a_{\sigma, j}(\varphi, \psi)=\left(A_{\sigma, j} \varphi, \psi\right), \quad \varphi, \psi \in H(K, \sigma) .
$$

Therefore, we have obtained the relation

$$
(\hat{f}(\lambda, \sigma) \varphi, \psi)=\left(\sum_{j=0}^{N}\left(A_{\sigma, j} \varphi, \psi\right) \lambda^{j}\right) e^{-t \lambda^{2}}
$$

for every $\varphi, \psi \in H(K, \sigma)$. This simply means that

$$
\hat{f}(\lambda, \sigma)=e^{-t \lambda^{2}} \sum_{j=0}^{N} \lambda^{j} A_{\sigma, j}
$$

as operators acting on $H(K, \sigma)$. From the definition of $\hat{f}(\lambda, \sigma)$ it follows that

$$
\sum_{j=0}^{N} \lambda^{j} A_{\sigma, j} \varphi(k)=e^{t \lambda^{2}} \int_{M(n)} f(x, u) e^{i \lambda k^{-1} e_{1} \cdot x} \varphi(k u) d x d u .
$$

We can calculate $A_{\sigma, j} \varphi(k)$ by taking derivatives on both sides at $\lambda=0: j ! A_{\sigma, j} \varphi(k)$ is the $j$-th derivative of the right hand side of (3.13) at $\lambda=0$. 
This shows that $A_{\sigma, j} \varphi$ is a finite linear combination of functions of the form

$$
\int_{M(n)} f(x, u)\left(k^{-1} e_{1} \cdot x\right)^{p} \varphi(k u) d x d u
$$

with $0 \leq p \leq j$. We can use Funk-Hecke formula to expand $\left(k^{-1} e_{1} \cdot x\right)^{p}=|x|^{p}\left(k^{-1} e_{1} \cdot x^{\prime}\right)^{p}$ in terms of spherical harmonics:

$$
\left(k^{-1} e_{1} \cdot x^{\prime}\right)^{p}=\sum_{m=0}^{p} \sum_{l=1}^{d_{m}} c_{m l} Y_{m l}\left(k^{-1} e_{1}\right) Y_{m l}\left(x^{\prime}\right)
$$

where the coefficients $c_{m l}$ are given by expanding $a(t)=t^{p}$ in terms of the ultraspherical polynomials. Therefore, $A_{\sigma, j} \varphi$ is a finite sum of terms of the form

$$
\begin{gathered}
\int_{M(n)} f(x, u)|x|^{p} Y_{m l}\left(x^{\prime}\right) Y_{m l}\left(k^{-1} e_{1}\right) \varphi(k u) d x d u \\
=Y_{m l}\left(k^{-1} e_{1}\right) \int_{K} f_{p, m, l}(u) \varphi(k u) d u
\end{gathered}
$$

where we have written

$$
f_{p, m, l}(u)=\int_{\boldsymbol{R}^{n}} f(x, u)|x|^{p} Y_{m, l}\left(x^{\prime}\right) d x .
$$

Finally, $e^{-t \lambda^{2}} \lambda^{j} A_{\sigma, j} \varphi(k)$ is a finite sum of functions of the form

$$
e^{-t \lambda^{2} \lambda^{j}} Y_{m, l}\left(k^{-1} e_{1}\right) \int_{K} f_{p, m, l}(u) \varphi(k u) d u
$$

In view of Lemma 3.1 this simply means that $\lambda^{j} e^{-t \lambda^{2}} A_{\sigma, j}$ is a finite sum of operators of the form $\hat{g}_{p, m, l}(\lambda, \sigma)$ with

$$
g_{p, m, l}(x, u)=|x|^{m} Y_{m, l}\left(x^{\prime}\right)\left(-\Delta_{n+2 m}\right)^{(j-m) / 2} p_{t}^{n+2 m}(x) f_{p, m, l}(u) .
$$

This completes the proof of Theorem 1.4.

We remark that when $N=0, s=t$ the conclusion of Theorem 1.4 takes the form

$$
f(x, u)=p_{t}^{n}(x)\left(\int_{\boldsymbol{R}^{n}} f(x, u) d x\right) .
$$

We now give a proof of Theorem 1.5, that is, a refined version of Hardy's theorem on $M(2)$. In this case it is slightly convenient to work with the following definition of $\hat{f}(\lambda)$ :

$$
\hat{f}(\lambda)=\int_{M(2)} f\left(z, e^{i \varphi}\right) \pi_{\lambda}\left(z, e^{i \varphi}\right)^{*} d z d \varphi
$$

where we use the complex variable $z$ to stand for elements of $\boldsymbol{R}^{2}$. An easy calculation shows that

$$
\hat{f}(\lambda) g(\theta)=\int_{0}^{2 \pi} \hat{f}\left(\lambda, e^{i(\theta+\varphi)}\right) g(\theta+\varphi) d \varphi
$$


where $g \in L^{2}(T)$ and

$$
\hat{f}\left(w, e^{i \varphi}\right)=\frac{1}{2 \pi} \int_{\boldsymbol{R}^{2}} e^{-i \operatorname{Re}(z \bar{w})} f\left(z, e^{i \varphi}\right) d z
$$

is the Euclidean Fourier transform of $f$ on $\boldsymbol{R}^{2}$.

From the above formula we obtain, with $e_{k}(\theta)=e^{i k \theta}$,

$$
\left(\hat{f}(\lambda) e_{k}, e_{j}\right)=\frac{1}{2 \pi} \int_{0}^{2 \pi} \int_{0}^{2 \pi} \hat{f}\left(\lambda e^{i \theta}, e^{i \varphi}\right) e^{i j \varphi} e^{i(k-j) \theta} d \theta d \varphi .
$$

Now, the Bessel functions $J_{n}(t)$ are given by the equation

$$
J_{n}(t)=\frac{1}{2 \pi} \int_{0}^{2 \pi} e^{-i t \sin \theta} e^{-i n \theta} d \theta .
$$

The functions $J_{n}(t)$ and $J_{-n}(t)$ are related by

$$
J_{n}(t)=(-1)^{n} J_{-n}(t) .
$$

In terms of the Bessel function we can write (see Rubin [9])

$$
\left(\hat{f}(\lambda) e_{k}, e_{j}\right)=\frac{(-i)^{k-j}}{2 \pi} \int_{0}^{2 \pi} \int_{0}^{2 \pi}\left(\int_{0}^{\infty} f\left(r e^{i \theta}, e^{i \varphi}\right) J_{k-j}(\lambda r) r d r\right) e^{i(k-j) \theta} e^{i j \varphi} d \theta d \varphi .
$$

If we define $\tilde{f}(r, m, n)$ to be the Fourier coefficients

$$
\tilde{f}(r, m, n)=\int_{0}^{2 \pi} \int_{0}^{2 \pi} f\left(r e^{i \theta}, e^{i \varphi}\right) e^{i m \theta} e^{i n \varphi} d \theta d \varphi
$$

then we can write the above as

$$
\left(\hat{f}(\lambda) e_{k}, e_{j}\right)=\frac{(-i)^{k-j}}{2 \pi} \int_{0}^{\infty} \tilde{f}(r, k-j, j) J_{k-j}(\lambda r) r d r .
$$

Assuming $k \geq j$ and defining

$$
g_{k j}(r)=\tilde{f}(r, k-j, j) r^{-k+j}
$$

we have the equation, with some constants $c_{k j}$,

$$
\left(\hat{f}(\lambda) e_{k}, e_{j}\right)=c_{k j} \lambda^{k-j} \int_{0}^{\infty} g_{k j}(r) \frac{J_{k-j}(\lambda r)}{(\lambda r)^{k-j}}(\lambda r)^{2(k-j)+1} d r .
$$

Thus, $\lambda^{-k+j}\left(\hat{f}(\lambda) e_{k}, e_{j}\right)$ is the Fourier transform of the radial function $g_{k j}(|x|)$ on $\boldsymbol{R}^{2(k-j)+2}$.

Now the conditions of the theorem on $f$ and $\hat{f}$ imply that

$$
\left|g_{k j}(|x|)\right| \leq c e^{-|x|^{2} / 4 t},\left|\hat{g}_{k j}(\xi)\right| \leq c e^{-t|\xi|^{2}}
$$

and hence by Hardy's theorem on $\boldsymbol{R}^{2(k-j)+2}$ we get $g_{k j}(r)=c_{k j} e^{-r^{2} / 4 t}$ which means

$$
\tilde{f}(r, k-j, j)=c_{k j} r^{k-j} e^{-r^{2} / 4 t} .
$$

When $k \neq j$ the equation (3.23) is not compatible with the estimate

$$
|\tilde{f}(r, k-j, j)| \leq c e^{-r^{2} / 4 t}
$$


unless $c_{k j}=0$. We can do the same thing when $k \leq j$ leading to the conclusion that

$$
\int_{0}^{2 \pi} \int_{0}^{2 \pi} f\left(r e^{i \theta}, e^{i \varphi}\right) e^{i m \theta} e^{i n \varphi} d \theta d \varphi=0
$$

whenever $m \neq 0$. We also have $\tilde{f}(r, 0, j)=c_{j} e^{-r^{2} / 4 t}$. Hence we obtain

$$
f\left(z, e^{i \varphi}\right)=p_{t}^{2}(z)\left(\sum_{j=-\infty}^{\infty} c_{j} e^{i j \varphi}\right) .
$$

This proves Theorem 1.5 with

$$
g\left(e^{i \varphi}\right)=\sum_{j=-\infty}^{\infty} c_{j} e^{i j \varphi}=\int_{R^{2}} f\left(z, e^{i \varphi}\right) d z .
$$

4. Beurling's theorem for $M(n)$. We begin a proof of Theorem 1.2 by obtaining an expression for the sum $\sum_{\sigma \in \hat{M}}\|\hat{f}(\lambda, \sigma)\|_{\mathrm{HS}}^{2}$. We make use of the unitary operator $U$ introduced in Section 2 to realise $\hat{f}(\lambda)$ as an integral operator on $L^{2}(K)$ whose kernel can be calculated. We use the explicit expression for this kernel to calculate the above sum.

As before let $\left\{Y_{m j} ; 1 \leq j \leq d_{m}, m=0,1,2, \ldots\right\}$ be an orthonormal basis of $L^{2}\left(S^{n-1}\right)$ consisting of spherical harmonics. Define

$$
f_{m j}(r, k)=\int_{S^{n-1}} f\left(r x^{\prime}, k\right) Y_{m j}\left(x^{\prime}\right) d x^{\prime}
$$

and let $g_{m j}(r, k)=r^{-m} f_{m j}(r, k)$. Also define

$$
\tilde{g}_{m j}(\lambda, k)=\int_{0}^{\infty} g_{m j}(r, k) \frac{J_{n / 2+m-1}(\lambda r)}{(\lambda r)^{n / 2+m-1}} r^{n+2 m-1} d r .
$$

Then we have the following result.

PROPOSITION 4.1.

$$
\sum_{\sigma \in \hat{M}}\|\hat{f}(\lambda, \sigma)\|_{\mathrm{HS}}^{2}=\sum_{m=0}^{\infty} \sum_{j=1}^{d_{m}} \lambda^{2 m} \int_{K}\left|\tilde{g}_{m j}(\lambda, k)\right|^{2} d k .
$$

ProOF. For $\lambda>0, \sigma \in \hat{M}$ and $\varphi, \psi \in H(K, \sigma)$

$$
\begin{aligned}
(\hat{f}(\lambda, \sigma) \varphi, \psi) & =\int_{M(n)} f(x, u)\left(\pi_{\lambda, \sigma}(x, u) \varphi, \psi\right) d x d u \\
& =\int_{M(n)} f(x, u)\left(\int_{K} e^{i \lambda\left(k^{-1} e_{1}, x\right)}(\varphi(k u), \psi(k)) d k\right) d x d u .
\end{aligned}
$$

We now make use of the expansion

$$
e^{i \lambda\left(k^{-1} e_{1}, x\right)}=\sum_{m=0}^{\infty} \sum_{j=1}^{d_{m}} \frac{J_{n / 2+m-1}(\lambda|x|)}{(\lambda|x|)^{n / 2-1}} Y_{m j}\left(k^{-1} e_{1}\right) Y_{m j}\left(x^{\prime}\right)
$$


where we have written $x=|x| x^{\prime}$. Using this expansion and recalling the definition of $\tilde{g}_{m j}(\lambda, u)$ we obtain

$$
\begin{aligned}
\hat{f}(\lambda, \sigma) \varphi(k) & =\int_{K}\left(\sum_{m=0}^{\infty} \sum_{j=1}^{d_{m}} \lambda^{m} \tilde{g}_{m j}(\lambda, u) Y_{m j}\left(k^{-1} e_{1}\right)\right) \varphi(k u) d u \\
& =\int_{K}\left(\sum_{m=0}^{\infty} \sum_{j=1}^{d_{m}} \lambda^{m} \tilde{g}_{m j}\left(\lambda, k^{-1} u\right) Y_{m j}\left(k^{-1} e_{1}\right)\right) \varphi(u) d u .
\end{aligned}
$$

Thus we have

$$
\hat{f}(\lambda, \sigma) \varphi(k)=\int_{K} G_{\lambda}(k, u) \varphi(u) d u
$$

where the kernel $G_{\lambda}$ is given by

$$
G_{\lambda}(k, u)=\sum_{m=0}^{\infty} \sum_{j=1}^{\infty} \lambda^{m} \tilde{g}_{m j}\left(\lambda, k^{-1} u\right) Y_{m j}\left(k^{-1} e_{1}\right) .
$$

Since $L^{2}(K, \sigma)$ is the direct sum of copies of $H(K, \sigma)$ the action of $\hat{f}(\lambda, \sigma)$ on $L^{2}(K, \sigma)$ is also given by

$$
\hat{f}(\lambda, \sigma) \varphi(k)=\int_{K} G_{\lambda}(k, u) \varphi(u) d u, \quad \varphi \in L^{2}(K, \sigma) .
$$

Using the unitary operator $U: L^{2}(K) \rightarrow L^{2}(K)^{\wedge}$ we consider the operator $U^{*} \hat{f}(\lambda) U$ on $L^{2}(K)$. For every $\psi \in L^{2}(K), U \psi(\sigma)(k)=\tilde{\psi}(\sigma, k)$ where

$$
\tilde{\psi}(\sigma, k)=\int_{M} \psi\left(u^{-1} k\right) \sigma(u) d u .
$$

By the definition of $\hat{f}(\lambda)$ we have

$$
\begin{aligned}
(\hat{f}(\lambda) U \psi)(\sigma)(k) & =\hat{f}(\lambda, \sigma) \tilde{\psi}(\sigma, k) \\
& =\int_{K} \int_{M} G_{\lambda}(k, u) \psi\left(m^{-1} u\right) \sigma(m) d m d u .
\end{aligned}
$$

If $T(\lambda, \sigma)=U^{*} \hat{f} U P_{\sigma}$ on $L^{2}(K)$ then from the definition of $P_{\sigma}$ and $U$ it follows that

$$
T(\lambda, \sigma) \psi(k)=\int_{K} \int_{M} G_{\lambda}(k, u) \psi\left(m^{-1} u\right) \operatorname{tr} \sigma(m) d m d u .
$$

With $\operatorname{tr} \sigma(m)=\chi_{\sigma}(m)$ we have

$$
T(\lambda, \sigma) \psi(k)=\int_{K}\left(\int_{M} G_{\lambda}(k, m u) \chi_{\sigma}(m) d m\right) \psi(u) d u .
$$

Since $L^{2}(K)$ is the direct sum of $P_{\sigma} L^{2}(K)$ as $\sigma$ ranges over $\hat{M}$ we see that

$$
\sum_{\sigma \in \hat{M}}\|T(\lambda, \sigma)\|_{\mathrm{HS}}^{2}=\left\|U^{*} \hat{f}(\lambda) U\right\|_{\mathrm{HS}}^{2}=\sum_{\sigma \in \hat{M}}\|\hat{f}(\lambda, \sigma)\|_{\mathrm{HS}}^{2} .
$$


Now $T(\lambda, \sigma)$ is an integral operator on $L^{2}(K)$ with kernel

$$
G_{\lambda, \sigma}(k, u)=\int_{M} G_{\lambda}(k, m u) \chi_{\sigma}(m) d m
$$

Therefore,

$$
\|T(\lambda, \sigma)\|_{\mathrm{HS}}^{2}=\int_{K} \int_{K}\left|G_{\lambda, \sigma}(k, u)\right|^{2} d k d u
$$

and summing over $\sigma \in \hat{M}$ we get

$$
\sum_{\sigma \in \hat{M}}\|\hat{f}(\lambda, \sigma)\|_{\mathrm{HS}}^{2}=\sum_{\sigma \in \hat{M}} \int_{K} \int_{K}\left|\int_{M} G_{\lambda}(k, m u) \chi_{\sigma}(m) d m\right|^{2} d k d u .
$$

Invoking Peter-Weyl we obtain

$$
\sum_{\sigma \in \hat{M}}\|\hat{f}(\lambda, \sigma)\|_{\mathrm{HS}}^{2}=\int_{K} \int_{K}\left|G_{\lambda}(k, u)\right|^{2} d k d u .
$$

Recalling the definition of $G_{\lambda}(k, u)$ and using the orthonormality of the spherical harmonics we complete the proof of the proposition.

Next we make the following observation. For $\delta \in \hat{K}$ consider the function $f * \chi_{\delta}$. Then writing $\pi_{\lambda, \sigma}(f)$ for $\hat{f}(\lambda, \sigma)$, an easy calculation shows that

$$
\pi_{\lambda, \sigma}\left(f * \chi_{\delta}\right)=\pi_{\lambda, \sigma}(f) \pi_{\lambda, \sigma}\left(\chi_{\delta}\right)
$$

Here the Fourier transform of $\chi_{\delta}$ is given by

$$
\begin{aligned}
\pi_{\lambda, \sigma}\left(\chi_{\delta}\right) \varphi(k) & =\int_{K} \chi_{\delta}(u) \pi_{\lambda, \sigma}(0, u) \varphi(k) d u \\
& =\int_{K} \chi_{\delta}(u) \varphi(k u) d u .
\end{aligned}
$$

Now $\rho(u)=\pi_{\lambda, \sigma}(0, u)$ is a representation of $K$ on $H(K, \sigma)$ and so $\rho$ is a direct sum of irreducible unitary representations of $K$. Let $\rho_{1}$ be a subrepresentation of $\rho$ realised on a subspace $V$ of $H(K, \sigma)$. Then on $V$

$$
\pi_{\lambda, \sigma}\left(\chi_{\delta}\right) \varphi(k)=\int_{K} \chi_{\delta}(u) \rho(u) \varphi(k) d u=\rho\left(\chi_{\delta}\right) \varphi(k) .
$$

But $\rho\left(\chi_{\delta}\right)=0$ unless $\rho$ is unitarily equivalent to $\delta$. Thus $\pi_{\lambda, \sigma}\left(\chi_{\delta}\right) \neq 0$ only when $\pi_{\lambda, \sigma}$ contains $\delta$. By Frobenius reciprocity, $\left[\pi_{\lambda, \sigma}, \delta\right]=[\delta, \sigma]$ (where $[\pi, \delta]$ is the multiplicity of $\delta$ in $\pi)$ and hence $\pi_{\lambda, \sigma}\left(\chi_{\delta}\right) \neq 0$ only when $[\delta, \sigma] \neq 0$. Thus $\pi_{\lambda, \sigma}\left(f * \chi_{\delta}\right) \neq 0$ only for finitely many $\sigma \in \hat{M}$ since $[\delta, \sigma]<\infty$. 
We now embark on a proof of Theorem 1.2. It is enough to show that $f * \chi_{\delta}=0$ for every $\delta \in \hat{K}$. If we let $f_{\delta}=f * \chi_{\delta}$ then $\pi_{\lambda, \sigma}\left(f_{\delta}\right) \neq 0$ only for finitely many $\sigma$ and hence

$$
\begin{gathered}
\int_{M(n)} \int_{0}^{\infty} \frac{\left(\sum_{\sigma \in \hat{M}}\left\|\hat{f}_{\delta}(\lambda, \sigma)\right\|_{\mathrm{HS}}^{2}\right)^{1 / 2}}{(1+\lambda)^{n}} \frac{\left|f_{\delta}(x, k)\right|}{(1+|x|)^{n}} e^{\lambda|x|} \lambda^{n-1} d \lambda d x d k \\
\leq \sum_{\sigma \in \hat{M}} \int_{M(n)} \int_{0}^{\infty} \frac{\left\|\hat{f}_{\delta}(\lambda, \sigma)\right\|_{\mathrm{HS}}}{(1+\lambda)^{n}} \frac{\left|f_{\delta}(x, k)\right|}{(1+|x|)^{n}} e^{\lambda|x|} \lambda^{n-1} d \lambda d x d k
\end{gathered}
$$

Since

$$
f_{\delta}(x, k)=\int_{K} f\left(x, k u^{-1}\right) \chi_{\delta}(u) d u=f * \chi_{\delta}(x, k)
$$

and $\hat{f}_{\delta}(\lambda, \sigma)=\hat{f}(\lambda, \sigma) \pi_{\lambda, \sigma}\left(\chi_{\delta}\right)$ the above is bounded by

$$
\sum_{\sigma \in \hat{M}} \int_{M(n)} \int_{0}^{\infty} \int_{K} \frac{\|\hat{f}(\lambda, \sigma)\|_{\mathrm{HS}}}{(1+\lambda)^{n}} \frac{\left|f\left(x, k u^{-1}\right)\right|}{(1+|x|)^{n}}\left|\chi_{\delta}(u)\right| e^{\lambda|x|} \lambda^{n-1} d \lambda d x d k d u
$$

which is finite by the hypothesis.

Appealing to the result of the proposition we conclude that for every $m \in N$ and $1 \leq$ $j \leq d_{m}$

$$
\int_{0}^{\infty} \int_{0}^{\infty} \int_{K} \lambda^{m} \frac{\left(\int_{K}\left|\tilde{g}_{m j}(\lambda, k)\right|^{2} d k\right)^{1 / 2}}{(1+\lambda)^{n}} \frac{\left|f_{m j}(r, k)\right|}{(1+r)^{n}} e^{\lambda r}(\lambda r)^{n-1} d r d \lambda d k<\infty
$$

where $f_{m j}$ and $g_{m j}$ are defined in terms of the function $f_{\delta}=f * \chi_{\delta}$. (We have suppressed the $\delta$-dependence for the sake of simplicity of notation). For each $\psi \in C(K)$ consider

$$
h_{m j}(r)=\int_{K} g_{m j}(r, k) \psi(k) d k
$$

so that the Hankel transform of type $(n / 2+m-1)$ of $h_{m j}(r)$ is

$$
\tilde{h}_{m j}(\lambda)=\int_{K} \tilde{g}_{m j}(\lambda, k) \psi(k) d k .
$$

Since $f_{m j}(r, k)=r^{m} g_{m j}(r, k)$ and

$$
\left|\tilde{h}_{m j}(\lambda)\right| \leq\left(\int_{K}\left|\tilde{g}_{m j}(\lambda, k)\right|^{2} d k\right)^{1 / 2}\|\psi\|_{2}
$$

we have

$$
\begin{aligned}
\int_{0}^{\infty} & \int_{0}^{\infty} \lambda^{m} \frac{\left|\tilde{h}_{m j}(\lambda)\right|}{(1+\lambda)^{n}} \frac{r^{m}\left|h_{m j}(r)\right|}{(1+r)^{n}} e^{\lambda r}(\lambda r)^{n-1} d r d \lambda \\
& \leq c \int_{0}^{\infty} \int_{0}^{\infty} \lambda^{m} \frac{\left(\int_{K}\left|\tilde{g}_{m j}(\lambda, k)\right|^{2} d k\right)^{1 / 2}}{(1+\lambda)^{n}} \frac{\left(\int_{K}\left|f_{m j}(r, k)\right| d k\right)}{(1+r)^{n}} e^{\lambda r}(r \lambda)^{n-1} d r d \lambda<\infty .
\end{aligned}
$$


Thus we have

$$
\int_{0}^{\infty} \int_{0}^{\infty} \frac{\left|h_{m j}(r)\right|}{(1+r)^{n+m}} \frac{\left|\tilde{h}_{m j}(\lambda)\right|}{(1+\lambda)^{n+m}} e^{\lambda r}(\lambda r)^{n+2 m-1} d r d \lambda<\infty .
$$

Since $\tilde{h}_{m j}(\lambda)$ is the Hankel transform of order $(n / 2+m-1)$ of $h_{m j}(r)$ we can appeal to Beurling's theorem on $\boldsymbol{R}^{n+2 m}$. Since $n+m \leq n+2 m$ we get $h_{m j}(r)=0$. As this is true for every $m$ and $j$ we get $f_{\delta}=0$. Hence $f=0$ as desired.

This completes the proof of Theorem 1.2. We now give a proof of Theorem 1.3.

We follow the same notation used in the proof of Theorem 1.5. Under the hypothesis of the theorem

$$
\begin{aligned}
& \int_{0}^{\infty} \int_{0}^{\infty} \frac{\left|\left(\hat{f}(\lambda) e_{k}, e_{j}\right)\right|}{(1+\lambda)^{3}} \frac{|\tilde{f}(r, k-j, j)|}{(1+r)^{3}} e^{\lambda r} \lambda r d r d \lambda \\
& \quad \leq c \int_{M(2)} \int_{0}^{\infty} \frac{\|\hat{f}(\lambda)\|_{\mathrm{HS}}}{(1+\lambda)^{3}} \frac{\left|f\left(z, e^{i \varphi}\right)\right|}{(1+|z|)^{3}} e^{\lambda|z|} \lambda d \lambda d z d \varphi<\infty .
\end{aligned}
$$

This means that, assuming $k \geq j$,

$$
\begin{aligned}
\int_{0}^{\infty} & \int_{0}^{\infty} \frac{\left|g_{k j}(r)\right| r^{(k-j)}}{(1+r)^{3}} \frac{\left|\tilde{g}_{k j}(\lambda)\right| \lambda^{(k-j)}}{(1+\lambda)^{3}} e^{\lambda r} \lambda r d r d \lambda \\
\quad & \int_{0}^{\infty} \int_{0}^{\infty} \frac{\left|g_{k j}(r)\right|}{(1+r)^{3+k-j}} \frac{\left|\tilde{g}_{k j}(\lambda)\right|}{(1+\lambda)^{3+k-j}} e^{\lambda r}(\lambda r)^{2(k-j)+1} d \lambda d r<\infty .
\end{aligned}
$$

Applying the result of Bonami et al. to the function $g_{k j}(|x|)$ on $\boldsymbol{R}^{2(k-j)+2}$ we obtain

$$
g_{k j}(|x|)=P_{k j}(x) e^{-|x|^{2} / 4 t_{k j}}
$$

where $\operatorname{deg} P_{k j} \leq 3+(k-j)-2(k-j)-3=-(k-j)$. Thus $P_{k j}=0$ unless $k=j$ and we have

$$
g_{k k}(|x|)=c_{k} e^{-|x|^{2} / 4 t_{k}}=\tilde{f}(|x|, 0, k)
$$

for some constants $c_{k}, t_{k}>0$. Therefore,

$$
f\left(z, e^{i \varphi}\right)=\sum c_{k} e^{i k \varphi} e^{-|z|^{2} / 4 t_{k}} .
$$

Now we recall that $\left(\hat{f}(\lambda) e_{k}, e_{j}\right) \lambda^{-(k-j)}$ is the Fourier transform of $\tilde{f}(r, k-j, j) r^{-(k-j)}$ on $\boldsymbol{R}^{2(k-j)+2}$. Therefore,

$$
\left(\hat{f}(\lambda) e_{k}, e_{j}\right)=\delta_{k j} c_{k} e^{-t_{k} \lambda^{2}}
$$

Hence, the equation

$$
\|\hat{f}(\lambda)\|_{\mathrm{HS}}^{2}=\sum_{k}\left\|\hat{f}(\lambda) e_{k}\right\|_{2}^{2}=\sum_{k, j}\left|\left(\hat{f}(\lambda) e_{k}, e_{j}\right)\right|^{2}
$$

gives us

$$
\|\hat{f}(\lambda)\|_{\mathrm{HS}}^{2}=\sum\left|c_{k}\right|^{2} e^{-2 t_{k} \lambda^{2}} .
$$


Thus the hypothesis on $f$ and $\hat{f}$ gives

$$
\int_{0}^{\infty} \int_{0}^{\infty} \frac{\|\hat{f}(\lambda)\|_{\mathrm{HS}}}{(1+\lambda)^{3}} \frac{e^{-r^{2} / 4 t_{k}}}{(1+r)^{3}} e^{\lambda r} \lambda r d r d \lambda<\infty .
$$

Since $\|\hat{f}(\lambda)\|_{\mathrm{HS}} \geq\left|c_{k}\right| e^{-t_{k} \lambda^{2}}$ for every $k$ we have

$$
\left|c_{j}\right| \int_{0}^{\infty} \int_{0}^{\infty} \frac{e^{-r^{2} / 4 t_{k}}}{(1+r)^{3}} e^{-t_{j} \lambda^{2}} e^{\lambda r} \lambda r d r d \lambda<\infty .
$$

It can be shown that the above is impossible unless $t_{k}=t_{j}$ for all $k$ and $j$. Thus $t_{k}=t$ for all $k$ and we have

$$
f\left(z, e^{i \varphi}\right)=e^{-|z|^{2} / 4 t}\left(\sum_{k} c_{k} e^{i k \varphi}\right)
$$

proving the theorem.

\section{REFERENCES}

[1] A. Bonami, B. Demange And P. Jaming, Hermite functions and uncertainty principles for the Fourier and windowed Fourier transforms, Rev. Mat. Iberoamericana 19 (2003), 23-55.

[2] M. Cowling, A. Sitaram And M. Sundari, Hardy's uncertainty principle on semisimple Lie groups, Pacific J. Math. 192 (2000), 293-296.

[3] M. Eguchi, S. Koizumi And K. Kumahara, An analogue of the Hardy theorem for the Cartan motion group, Proc. Japan Acad. Ser. A, Math. Sci. 74 (1998), 149-151.

[ 4 ] M. Eguchi, S. Koizumi And K. Kumahara, An $L^{p}$ version of the Hardy theorem for motion groups, J. Aust. Math. Soc. Ser. A, 68 (2000), 55-67.

[ 5 ] G. B. Folland, A course in abstract harmonic analysis, Stud. Adv. Math. CRC Press, Boca Raton, Fla, 1995.

[6] K. I. Gross And R. A. KunZE, Fourier decompositions of certain representations, Symmetric spaces (Short Courses, Washington Univ., St. Louis, Mo., 1969-1970), 119-139, Boothby and Weiss, Eds., MarcelDekker, New York, 1972.

[ 7 ] L. HöRMANDER, A uniqueness theorem of Beurling for Fourier transform pairs, Ark. Mat. 29 (1991), 237240.

[ 8 ] C. Muller, Spherical harmonics, Lecture Notes in Math. 17, Springer Verlag, Berlin-Heidelberg-New York, 1966.

[ 9 ] R. L. RuBin, Harmonic analysis on the group of rigid motions of the Euclidean plane, Studia Math. 62 (1978), 125-141.

[10] R. P. SARKAR, A complete analogue of Hardy's theorem on semisimple Lie groups, Colloq. Math. 93 (2002), $27-40$

[11] A. SitARAm And M. Sundari, An analogue of Hardy's theorem for very rapidly decreasing functions on semisimple groups, Pacific J. Math. 177 (1997), 187-200.

[12] E. M. Stein And G. Weiss, Introduction to Fourier analysis on Euclidean spaces, Princeton Univ. Press, Princeton, N.J., 1971.

[13] M. Sugiura, Unitary representations and harmonic analysis, Halsted Press, New York-London-Sydney, 1975.

[14] M. Sundari, Hardy's theorem for the $n$-dimensional Euclidean motion group, Proc. Amer. Math. Soc. 126 (1998), 1199-1204.

[15] S. Thangavelu, Revisiting Hardy's theorem for the Heisenberg group, Math. Z. 2424 (2002) 761-779.

[16] S. Thangavelu, Hardy's theorem for the Helgason Fourier transform on noncompact rank one symmetric spaces, Colloq. Math. 94 (2002), 263-280. 
Stat-Math Division

INDIAN STATISTICAL INSTITUTE

203 B.T. RD

CALCUTTA 700108

INDIA

E-mail address: rudra@isical.ac.in
Stat-Math Division

INDIAN STATISTICAL INSTITUTE

8 TH MiLe, Mysore RD

BANGALORE 560059

INDIA

E-mail address: veluma@isibang.ac.in 